Eur Neurol 1996;36:107-108

\begin{tabular}{|l|l|l|}
\hline H.-H. & Horng-Huei & Liou $^{\text {a }}$ \\
\hline H.-C. & Hou-Chang & Chiub $^{\text {a,c }}$ \\
\hline H.-M. & Hon-Man & Liu $^{b}$ \\
\hline
\end{tabular}

${ }^{a}$ Departments of Neurology and ${ }^{\mathrm{b}}$ Radiology, National Taiwan University, Hospital, and ${ }^{\mathrm{C} D e p a r t m e n t ~ o f ~ N e u r o l o g y, ~ S h i n ~ K o n g ~ W u ~ H o-S u, ~}$ Memorial Hospital, Taipei, Taiwan

\title{
Abnormal Enhancement of the Left Putamen on Brain MRI in a Case of Proven Creutzfeldt-Jakob \\ Disease
}

Dr. Horng-Huei Liou, MD, Department of Neurology, National Taiwan University Hospital, No. 7 Chung-Shan South Road, Taipei 100 (Taiwan)

Creutzfeldt-Jakob disease (CJD) is caused by unusual protein-aceous infectious particles, or prions [1]. Criteria for the clinically definite diagnosis of CJD include rapid-evolving dementia, myoclonus, periodic electroencephalographic activity (1-2 Hz) and finally death within 12 months [2]. Magnetic resonance imaging (MRI) of CJD cases show increased signal intensity in the bilateral basal ganglia on T2-weighted images [3-6]. We report here a case of pathologically proven CJD, showing abnormal enhancement in left putamen on godolinum-diethylenetriamine pentaacetic acid (Gd-DTPA) $\mathrm{T}_{\mathrm{r}}$ weighted MRI (T1WI), which has not been reported before.

A 52-year-old male truck driver became progressively forgetful and aphasic over a 6-week period. He then gradually developed dressing and gait apraxia; urinary incontinence and visual hallucinations were also reported. Startle myoclonus induced by auditory or visual stimulation and spontaneous generalized myoclonus or focal seizure were seen during this period. He became bedridden and detached from others 10 weeks later. On examination 11 weeks after

the onset of symptoms, the patient was in a vegetative state with stable vital signs. Neurological examination revealed preserved brain stem reflexes and obvious primitive reflexes. There was generalized hyperreflexia with bilateral ankle clonus and Babinski signs. No focal weakness was detected. In addition, he had episodic generalized myoclonus, generalized tonic-clonic seizures and focal seizures, which occurred separately. Normal values were reported for the blood cell count, chemistry profile, thyroid function, tumor marker screen, ceruloplasmin, and lumbar puncture. Immunological profiles, serologic test for syphilis and screening for AIDS were also normal. EEG on admission showed generalized continuous periodic sharp-wave complexes with an interval of 1-2 s. Two weeks later, EEG showed almost continuous periodic generalized stereotypic sharp waves, with a recurrent rate of $0.5-0.6 \mathrm{~Hz}$, and short periods of suppression burst pattern. Computerized tomography, 12 weeks after onset, was unremarkable. MRI (1.5 T, Signa, General Electric), 14 weeks after onset, showed multiple abnormal signal lesions bilaterally in the putamen, caudate nucleus, and hippocampus. These appeared bright on the $\mathrm{T}_{2}$-weighted images (T2WI) (fig. 1), and the lesion in the inferior aspect of the left putamen showed abnormal enhancement using Gd-DTPA (fig. 2). The ventricles and sulci were dilated. No mass effect was seen. A biopsy of the right frontal lobe, performed 3 months after onset, showed cerebral atrophy. Microscopically, diffuse neuronal loss, striking vacuolation, and hypertro-phied astrocytes of the gray matter were seen. No known mutation of the prion protein (Prp) gene [7] was found in the DNA analysis of either the patient or his family members. In spite of intensive treatment, the patient died 4.5 months after the onset of symptoms.

of the prion protein (Prp) gene [7] was found in the
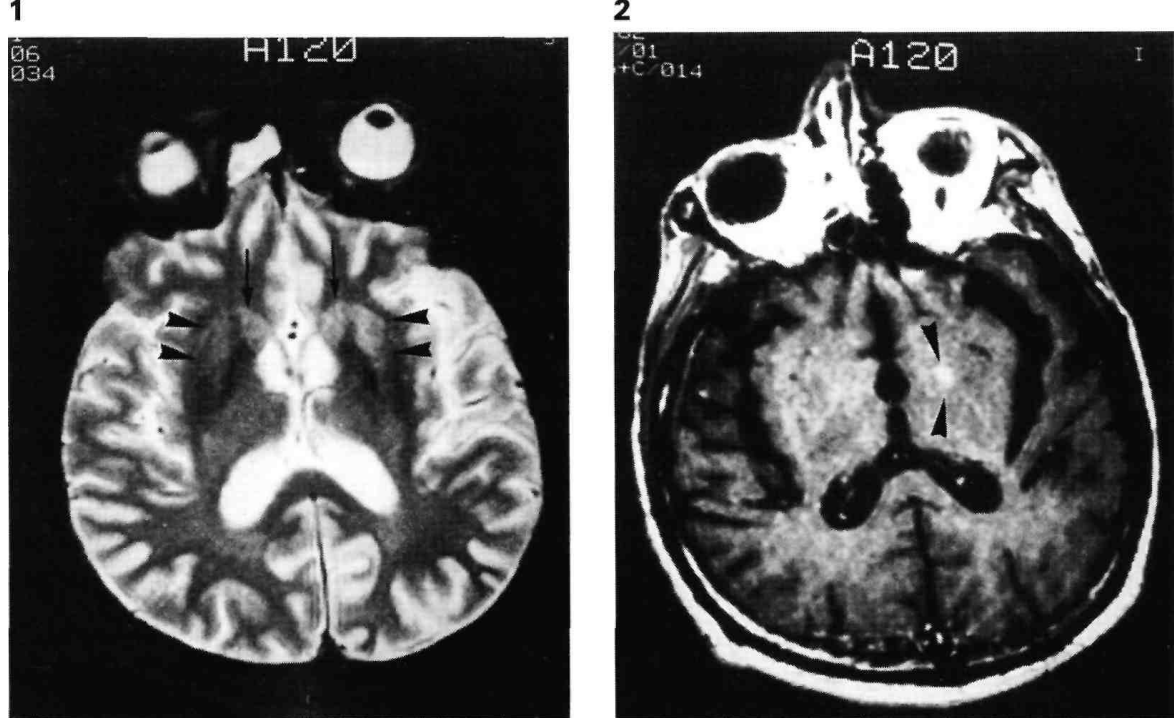

Fig. 1 . Axial T2WI $(\mathrm{TR} / \mathrm{TE}=2,200 \mathrm{~ms} / 100 \mathrm{~m}$
The ventricles and sulci were slightly dilated.

Fig. 2. Axial IR images (TR/TI/TE $=1,800 \mathrm{~ms} / 830 \mathrm{~ms} / 20 \mathrm{~ms}$ ) after Gd-DTPA enhancement. Abnormal enhancement was shown in the inferior aspect of the left putamen (arrowheads).

\section{KAHGEII}

E-Mail karger@karger.ch Fax + 41613061234

107

Our patient showed typical clinical and pathological features of CJD [2]. On DNA analysis, known mutations of the Prp gene were not found in our patient or his family [8], suggesting that he may be a case of sporadic CJD. MRI study of CJD may show increased signal intensity bilaterally in the head of the caudate, putamen, globus palli-dus, thalamus, and cerebral cortex on T2WI [3-7]. However, only cortical atrophy with no apparent white-matter changes has also been reported [9]. Milton et al. [4] stated that in a patient with rapid progressive dementia, the MRI demonstration of increased bilateral signal intensity on long repetition time images in deep gray structures should suggest the diagnosis of CJD. In the different stages of CJD, deterioration of the clinical condition correlates with basal ganglia and periventricular hyperintensity on T2WI [5]. This suggests that white-matter involvement in CJD may develop later upon degeneration of the cortex and subcortical gray matter [5]. Our case showed increased signal intensity in the bilateral head of the caudate and putamen on T2WI. Enhancement by Gd-DTPA on T1WI was also seen in the left putamen, which has not been noted in previous reports [3-7]. Brain biopsy revealed no inflammatory changes and MRI showed no mass effect. Therefore, the enhancement is neither related to an inflammatory nor to a neoplastic process. Since gliosis and nerve cell loss occur later in the disease process than spongiform changes [10], the former are probably more important in causing this reported MRI changes in CJD. We presume that in our case this enhancement might have been due to excessive astrogliosis.

In conclusion, our case not only confirmed previous MRI studies, but also showed Gd-DTPA T1WI enhancement in the left putamen, which occurred 3 weeks before his death. These MRI findings might be explained by astrogliosis in an advanced stage of CJD

References

Prusiner SB: Novel proteinaceous infectious particles cause scrapie. Science 1982;216:136-144. 
Brown P, Cathala F, Castaigne P, Gajdusek DC: Creutzfeldt-Jakob disease: Clinical analysis of a consecutive series of 230 neuropathologically verified cases. Ann Neurol 1986;20:597-602. Gertz HJ, Henks H, Cervos-Navarro JC: Creutzfeldt-Jakob disease: Correlation of MRI and neuropathic findings. Neurology 1988;38:1481-1482.

Milton WJ, Atlas SW, Lavi E, Mollman JE: Magnetic resonance imaging of Creutzfeldt-Jakob disease. Ann Neurol 1991;29:438-440.

Yamamoto K, Morimatsu M: Increased signal in basal ganglia and white

Neuroll1992;29:114.

Neurollig92;29:114.

Tartaro A, Fulgente T, Pizzi CD, Bonomo L, Bocola V, Onofrj M: MRI alterations as an early finding in Creutzfeldt-Jakob disease. Eur $J$ Radio 1993;17:155-158.

Johns DW, Drazkowski JF, Draycr BP, Lieberman AN: Appearance of striatal signal hyperintensity on MRI preceding classic symptoms in ataxic Creutzeldt-Jakob disease: Case report. BNI Q 1993;9:14-17.

Calmer MS, Dryden AJ, Hughes J, Collinge J. Homozygous prion protei 352:340-342.

Kovanen J, E Cinjuti T, Iivan onch M, Ketonen L, Haltia M, Sulkava R, Sipponen, JT: Ceno Sipponen JT: Cerebral MR and CT imaging in Creutzfeldt-Jakob disease. J
Comput|Assist Tomogr 1985;9:125-128.

(Creact (Crentelit-Jakob disease) The nut

change. Brain 1978;101:333-344. 